\title{
Application of Classification Methods to Individual Disability Income Insurance Fraud Detection
}

\author{
Yi Peng ${ }^{1}$, Gang Kou ${ }^{1,3, *}$, Alan Sabatka ${ }^{2}$, Jeff Matza ${ }^{2}$, Zhengxin Chen ${ }^{1}$, \\ Deepak Khazanchi ${ }^{1}$, and Yong $\mathrm{Shi}^{1}$ \\ ${ }^{1}$ Peter Kiewit Institute of Information Science, Technology \& Engineering, University of \\ Nebraska, Omaha, NE 68182, USA \\ ${ }^{2}$ Mutual of Omaha, Omaha, NE, USA \\ ${ }^{3}$ Thomson Co., R\&D, 610 Opperman Drive, Eagan, MN 55123, USA \\ Tel.: ++1 4024030269 \\ kougang@yahoo.com
}

\begin{abstract}
As the number of electronic insurance claims increases each year, it is difficult to detect insurance fraud in a timely manner by manual methods alone. The objective of this study is to use classification modeling techniques to identify suspicious policies to assist manual inspections. The predictive models can label high-risk policies and help investigators to focus on suspicious records and accelerate the claim-handling process.

The study uses health insurance data with some known suspicious and normal policies. These known policies are used to train the predictive models. Missing values and irrelevant variables are removed before building predictive models. Three predictive models: Naïve Bayes (NB), decision tree, and Multiple Criteria Linear Programming (MCLP), are trained using the claim data. Experimental study shows that NB outperformed decision tree and MCLP in terms of classification accuracy.
\end{abstract}

Keywords: Classification, Insurance Fraud Detection, Naïve Bayes (NB), decision tree, and Multiple Criteria Linear Programming (MCLP).

\section{Introduction}

Health care frauds cost private health insurance companies and public health insurance programs at least $\$ 51$ billion in calendar year 2003 (The National Health Care Anti-Fraud Association, 2005). Traditional heuristic-rule based fraud detection techniques can not identify complex fraud schemes. Such a situation demands more sophisticated analytical methods and techniques that are capable of detecting fraud activities from large databases.

The objective of this study is to design, develop and test classification models to detect abnormal behaviors in disability income insurance data. The predictive models

* The corresponding author. 
can label high-risk policies and help investigators to focus on suspicious records and accelerate the claim-handling process. To achieve the goal, this project studies the attributes; preprocesses dataset; and develops classification models using a three-step approach. The first step is model construction. A predictive model is constructed based on training dataset, which includes predefined class labels for each record. The second step is model validation. This step uses validation dataset to tune the model weights during estimation and assess the classification accuracy of the model. The third step is model usage. The classification model developed and validated in the first and second step is used to classify future unknown data into predefined classes. For comparison purposes, three classifiers: Naïve Bayes (NB), decision tree, and Multiple Criteria Linear Programming (MCLP), are constructed for the dataset. The resultant classification model(s) should provide acceptable classification accuracies and allow domain experts to use the classification model in future detection of abnormal behaviors.

This paper is organized as follows: The next section describes data and preprocessing steps, including feature selection and data transformation. The third section gives an overview of the three classification techniques. The fourth section presents the classification results and compares the three classifiers. The last section concludes the paper with future research direction.

\section{Data Understanding and Data Preparation}

The insurance data are from Mutual of Omaha insurance company (Mutual of Omaha, 2006). There are five datasets that provide information about policy types, claims, producers, and clients. The attributes include numeric, categorical, and date types. These datasets were combined into one dataset using a common unique key. The final dataset has over 18,000 records with 103 variables. A target attribute indicates the class of each record in the final dataset. These records belong to either Normal or Abnormal class.

Data preparation removes irrelevant variables and missing values; conducts correlation analysis to understand the relationship between attributes and the target attribute; selects attributes for classification modeling; and transforms attributes to appropriate forms for the three classifiers. If all instances of a variable are missing, this variable has no use in the classification and was removed.

Correlation analysis describes the relation between two or more variables. The goal of correlation analysis is to find out which variables are strongly associated with the target variable. Since many variables are categorical, Cramer's V, a popular chisquare-based measure of nominal association, was used. Cramer's V ranges from 0 to 1. The closer Cramer's V is to 0, the smaller association between the two variables. The closer $\mathrm{V}$ is to 1 , the stronger association between the two variables. Attributes with $\mathrm{V}>0.1$ were selected for classification. As a result, the number of attributes was reduced from 103 to 20 .

The software tools used in this study are base SAS, SAS Enterprise Miner (EM), and a $\mathrm{C}++$ program for MCLP. Naïve Bayes (NB), decision tree, and MCLP require 
different data types. The tree node of SAS EM handles the data transformation for decision tree method. NB requires attributes to be categorical while MCLP requires attributes to be numeric. Data transformation for NB and MCLP were carried out by base SAS and $\mathrm{C}++$.

\section{Classification Techniques}

Based on the available data, three classification methods -- decision tree, Naïve Bayes, and Multiple Criteria Linear Programming (MCLP), were proposed. They classify data from different perspectives.

Decision tree is a machine learning technique that applies a divide-and-conquer method to recursively build a tree to classify data records. It is one of the most popular classification tools due to its high classification accuracy and easily interpretable results. From our previous experience, decision tree is efficient and superior to many other classifiers for different data sets. SAS EM provides tree nodes that compute decision tree solution.

Naïve Bayes (NB) classifier is a simple probabilistic classifier that computes the probabilities of instances belonging to each predefined class and assigns instances to the class that has the highest probability. NB is proposed for this project for two reasons. First, many variables selected by correlation analysis are categorical and NB is designed to classify categorical data. Second, despite its naïve design, NB works well in many real-world situations and outperforms some complex classifiers. In addition, NB takes approaches that are different from decision tree and MCLP to build classifier. NB function was implemented in base SAS.

MCLP is a mathematical-based classification approach that classifies records into distinct groups based on two or more objective functions. Previous experiences (Peng et al 2004; Kou et al 2005) proved that MCLP achieves comparable or superior classification results than other popular classification methods for various datasets. A self-developed C++ program (Kou et al 2003) was applied in this study to construct and solve the MCLP model.

\section{Empirical Study}

\subsection{Decision Tree}

The Tree node in SAS EM provides three criteria for tree splitting: chi-square test, entropy reduction, and gini reduction. Each criterion uses different techniques to split tree and establish a different decision tree model. Since each technique has its own bias, it may potentially provide a stronger solution if the results from three models are integrated. The method of integrating results from multiple models is called ensemble in machine learning. The following tables summarize the output of four decision tree models: chi-square test, entropy reduction, gini reduction, and ensemble. For each model, the confusion matrix is reported. 
Table 1. Decision Tree Model using chi-square test

\begin{tabular}{|c|c|c|c|}
\hline \multicolumn{4}{|c|}{ Confusion Matrix } \\
\hline & Predict & & \\
\hline Actual & 1 & 2 & Total \\
\hline 1 & 18510 & 12 & 18522 \\
\hline 2 & 316 & 37 & 353 \\
\hline Total & 18826 & 49 & 18875 \\
\hline
\end{tabular}

$18510\left(2^{\text {nd }}\right.$ row, $2^{\text {nd }}$ column) is the correct predictions that a record is class $1-$ Normal.

$12\left(2^{\text {nd }}\right.$ row, $3^{\text {rd }}$ column $)$ is the incorrect predictions that a record is class 2 Abnormal.

316 ( $3^{\text {rd }}$ row, $2^{\text {nd }}$ column $)$ is the incorrect predictions that a record is class $1-$ Normal. 37 ( $3^{\text {rd }}$ row, $3^{\text {rd }}$ column $)$ is the correct predictions that a record is class 2 - Abnormal.

Table 2. Decision Tree Model using entropy reduction

\begin{tabular}{|l|l|l|l|}
\multicolumn{3}{c}{ Confusion Matrix } \\
\hline \multicolumn{3}{|l|}{ Predicted } & \\
\hline Actual & 1 & 2 & Total \\
\hline 1 & 18458 & 64 & 18522 \\
\hline 2 & 102 & 251 & 353 \\
\hline Total & 18560 & 315 & 18875 \\
\hline
\end{tabular}

Table 3. Decision Tree Model using gini reduction

Confusion Matrix

\begin{tabular}{|c|c|c|c|}
\hline & \multicolumn{2}{|c|}{ Predicted } & \\
\hline Actual & 1 & 2 & Total \\
\hline 1 & 18496 & 26 & 18522 \\
\hline 2 & 213 & 140 & 353 \\
\hline Total & 18709 & 166 & 18875 \\
\hline
\end{tabular}

Table 4. Ensemble: chi-square, entropy reduction, and gini reduction

Confusion Matrix

\begin{tabular}{|l|l|l|l|}
\hline & \multicolumn{2}{|l|}{ Predicted } & \\
\hline Actual & 1 & 2 & Total \\
\hline 1 & 18519 & 3 & 18522 \\
\hline 2 & 227 & 126 & 353 \\
\hline Total & 18746 & 129 & 18875 \\
\hline
\end{tabular}

The ensemble node creates a new model by averaging the posterior probabilities from chi-square, entropy, and gini models. 
All decision tree results, except the one using entropy reduction, have less than $50 \%$ classification accuracy for class 2 records. Since the prediction accuracy of class 2 (Abnormal class) is important in fraud detection, decision tree is not a satisfactory solution for this dataset.

Table 5. Naïve Bayes

Confusion Matrix

Training: $\mathbf{4 0 \%}$

\begin{tabular}{|c|c|c|c|}
\hline & \multicolumn{2}{|c|}{ Predicted } & \\
\hline Actual & 1 & 2 & Total \\
\hline 1 & 7272 & 134 & 7406 \\
\hline 2 & 1 & 143 & 144 \\
\hline Total & 7273 & 277 & 7550 \\
\hline
\end{tabular}

Test: $\mathbf{3 0 \%}$

\begin{tabular}{|c|c|c|c|}
\hline & \multicolumn{2}{|c|}{ Predicted } & \\
\hline Actual & 1 & 2 & Total \\
\hline 1 & 4907 & $\mathbf{6 5 6}$ & 5563 \\
\hline 2 & 42 & 58 & 100 \\
\hline Total & 4949 & 714 & 5663 \\
\hline
\end{tabular}

All data

\begin{tabular}{|c|c|c|c|}
\hline & \multicolumn{2}{|c|}{ Predicted } & \\
\hline Actual & 1 & 2 & Total \\
\hline 1 & 17949 & $\mathbf{5 7 3}$ & 18522 \\
\hline 2 & 2 & 351 & 353 \\
\hline Total & 17951 & 924 & 18875 \\
\hline
\end{tabular}

NB produces much higher class 2 classification accuracy than decision tree. For test data, NB achieves 99\% classification accuracy for class 2 records, while the highest result of decision tree (using entropy reduction) is only $71 \%$.

\subsection{Multiple Criteria Linear Programming (MCLP)}

MCLP requires all variables to be numeric, which is contrary to NB. The following tables illustrate the training and test results of MCLP. The overall classification accuracies for training and test are 59\% and 57.95\%, respectively.

The results of NB, Decision Tree, and MCLP indicate that probability-based methods, such as NB, outperform decision tree and MCLP for this dataset. The reason may be that many variables that are strongly associated with the target variable are categorical. When these variables were transformed into numerical types, part of the information is lost. 
Table 6.

\section{Training}

\begin{tabular}{|c|c|r|r|c|}
\hline & \multicolumn{2}{|c|}{ Predicted } & & \\
\hline Actual & 1 & 2 & Total & $\begin{array}{c}\text { Corr } \\
\text { Rate }\end{array}$ \\
\hline 1 & 61 & 39 & 100 & $61 \%$ \\
\hline 2 & 43 & 57 & 100 & $57 \%$ \\
\hline Total & 104 & 96 & 200 & $59 \%$ \\
\hline
\end{tabular}

Test

\begin{tabular}{|c|c|r|c|r|}
\hline & \multicolumn{2}{|c|}{ Predicted } & & \\
\hline Actual & 1 & 2 & Total & Corr Rate \\
\hline 1 & 143 & 110 & 253 & $56.52 \%$ \\
\hline 2 & 7743 & 10679 & 18422 & $57.97 \%$ \\
\hline Total & 7886 & 10789 & 18675 & $57.95 \%$ \\
\hline
\end{tabular}

\section{Directions for Future Projects}

Based on the results of this project, we propose several future directions. The first direction is to further validate the classification classifier with new data. The second direction is to update the existing labeled data and classifiers. A fundamental job of a classifier is to identify patterns in unknown data that are similar to those in known data. The quality of known or training data determines the classification results. Labeled data need to be updated periodically because data patterns change over time. Accordingly, classifiers need to be trained periodically to catch the changes in the data. The third direction is to try different classification methods that can handle categorical data and compare the results with NB. The last suggestion is to try oneclass classification algorithm. Normal classification needs two or more classes, while one-class classification only needs information of the target class. One-class classification is useful when labeled target class records are available. By concentrating on target class data, one-class classification may generate better results.

\section{Acknowledgment}

This research has been supported by "Data Mining Project - Individual Disability Income Product", Mutual of Omaha. 


\section{References}

1. The National Health Care Anti-Fraud Association, available at: http://www.nhcaa.org/ (2005)

2. Kou, G., Liu, X., Peng, Y., Shi, Y., Wise, M., Xu, W.: Multiple Criteria Linear Programming to Data Mining: Models, Algorithm Designs and Software Developments Optimization Methods and Software 18 (4):, Part 2 AUG (2003) 453-473

3. Kou, G., Peng, Y., Shi, Y., Wise, M., Xu, W.: Discovering Credit Cardholders' Behavior by Multiple Criteria Linear Programming Annals of Operations Research 135 (1):, JAN (2005) 261-274

4. Mutual of Omaha Website: http://www.mutualofomaha.com/. (2006)

5. Peng, Y., Kou, G., Chen, Z. and Shi, Y.: Cross-validation and ensemble analyses on multiple-criteria linear programming classification for credit cardholder behavior in Bubak M et al, eds., ICCS 2004, LNCS 3039:, Krakow, POLAND, JUN 06-09, (2004) 931-939 